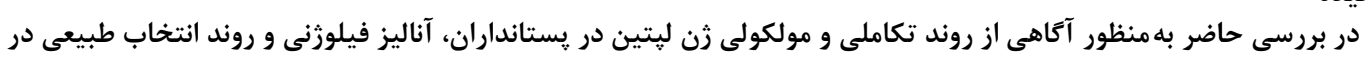

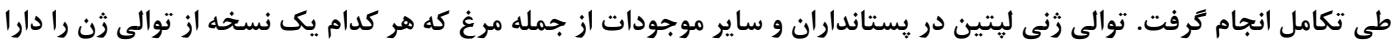

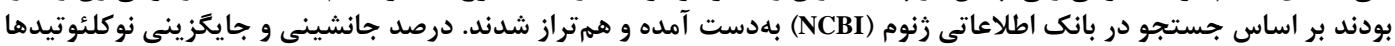

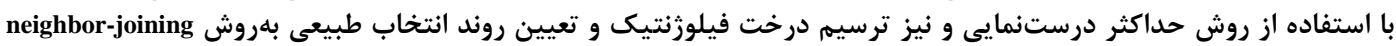

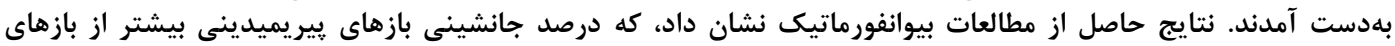

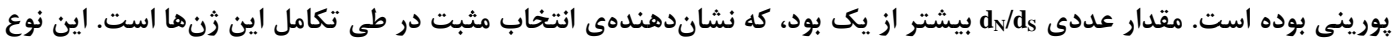

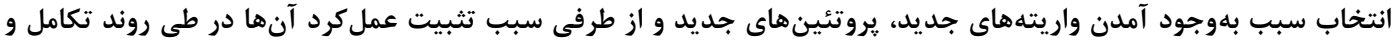

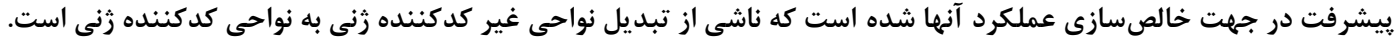

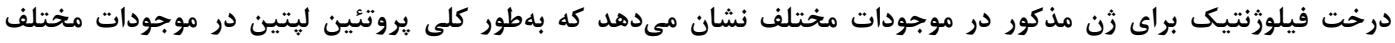

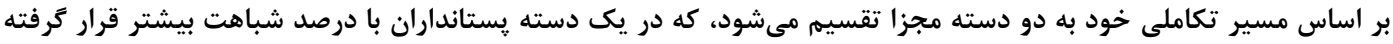

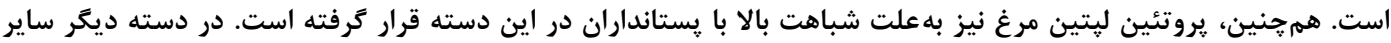

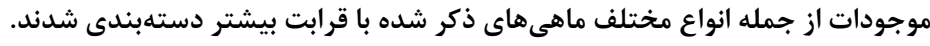

وازههاى كليدى: ليتين، پِتانداران، انتخاب طبيعى، فيلوزنى

احيا كردن روابط تكاملى بين گونههاى مختلف و برآورد

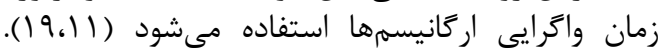

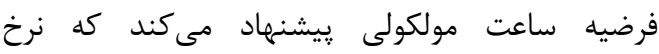

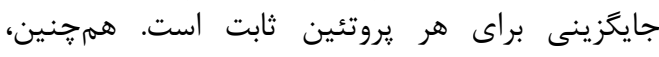

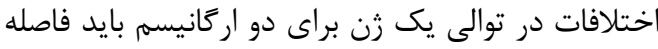

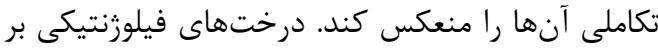

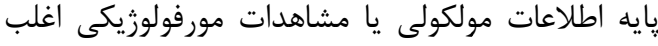

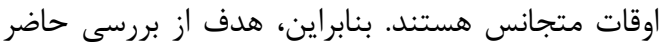

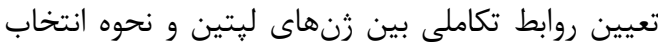

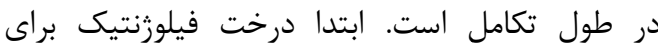

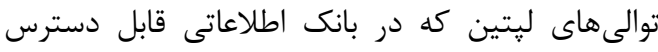

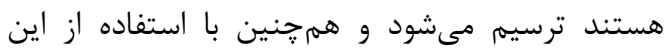

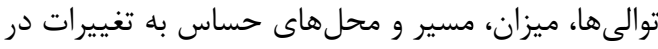

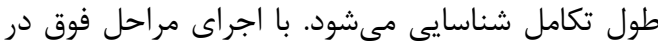

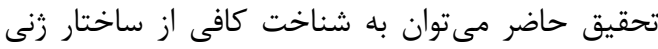

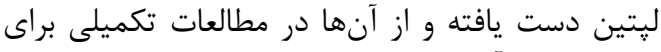

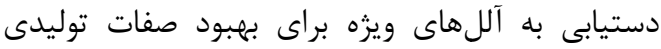
استفاده كرد.
مقدمه

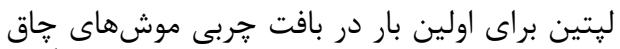

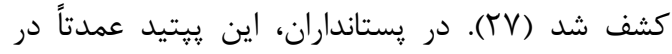

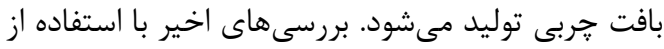

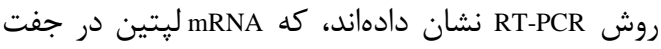
(Y، (Y)

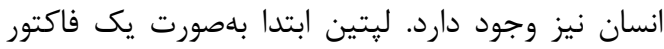

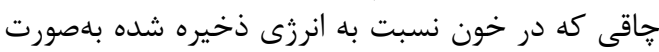

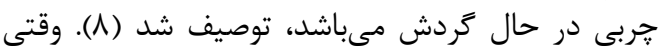

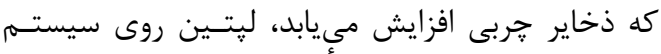

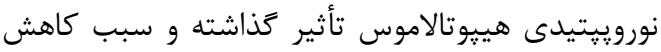

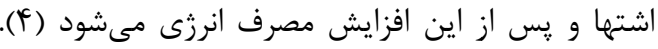

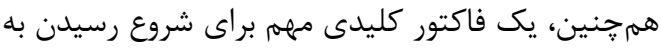

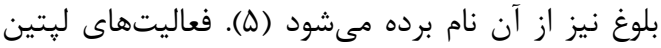

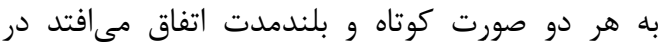

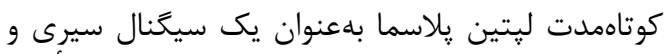

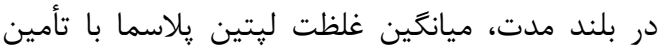

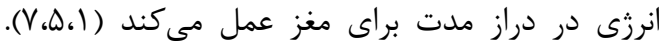
فيلوزنى مولكولى از اطلاعات توالى دراى مولى مولكولى براى مئى 
$\mathrm{d}_{\mathrm{S}}=-3 / 4 \operatorname{Ln}\left(1-4 / 3 \mathrm{P}_{\mathrm{S}}\right)$

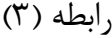

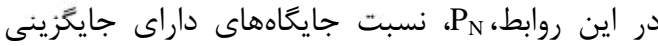

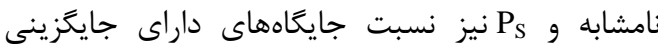

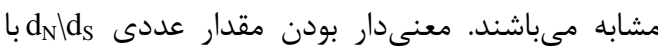

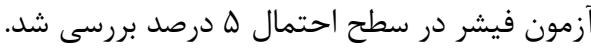

\section{نتايج و بحث}

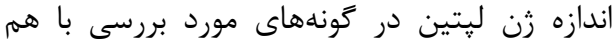

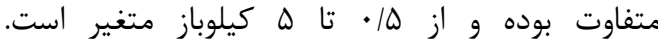

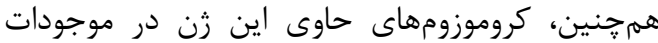

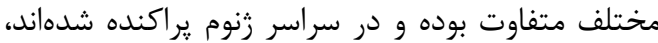

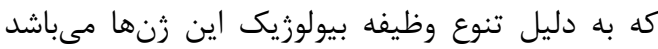

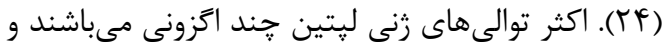

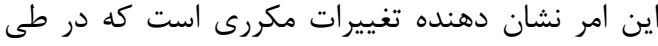

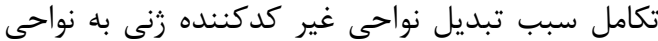

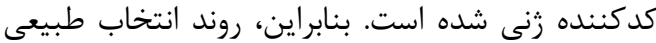

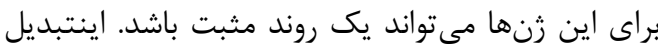

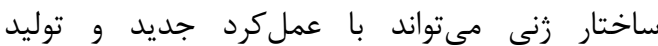

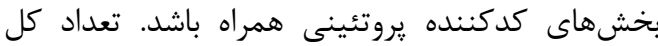

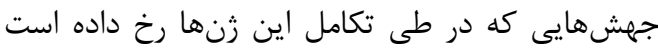

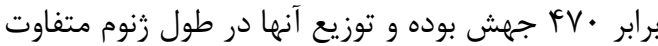

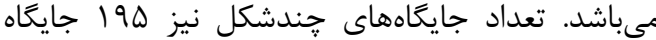

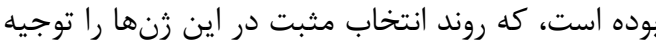

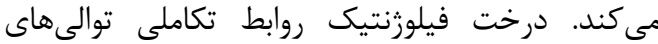

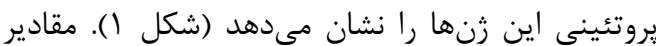

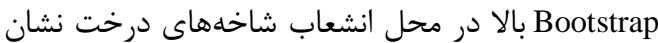

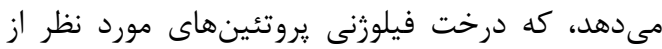
درجه اعتماد بالايى برخوردار است فيل

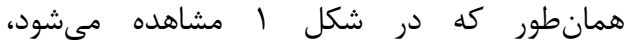

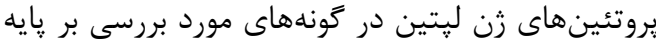

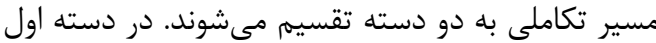

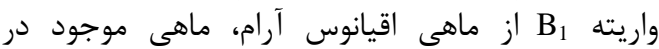
اقيانوس اطلس، ماهى زإينى و نيز إنيز واريته

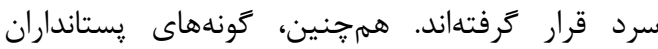

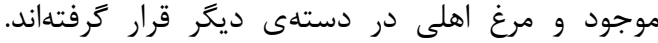

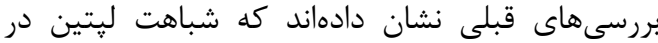

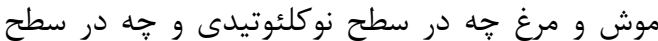

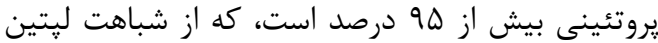

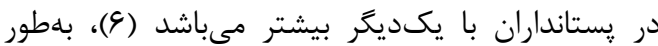

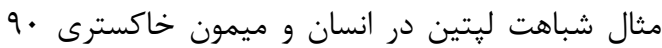

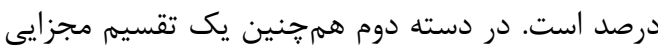

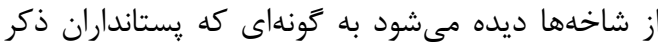

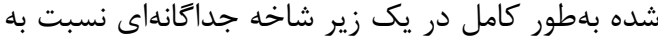

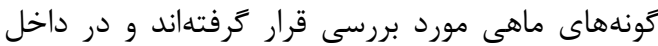

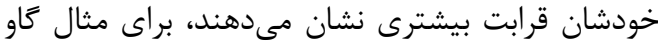

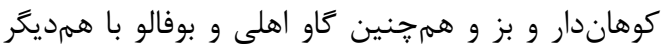
قرابت بيشترى دارند و اين روند براى سائ ساير پِّتانداران

1- National Center for Biotechnology Information 3- Neighbor-Joining

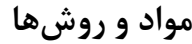
شناسايى و ويرايش توال هاى رنى ليتين

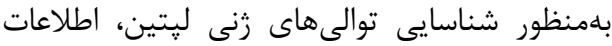

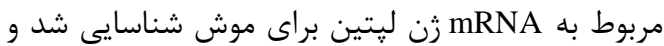

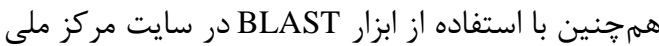

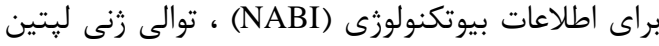

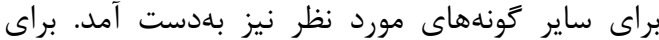

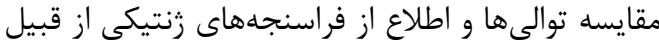

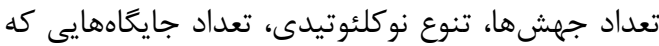

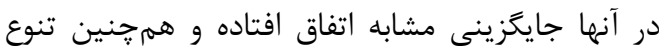

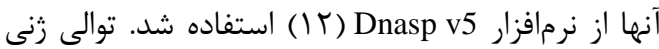

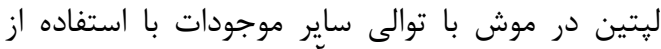

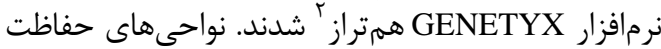

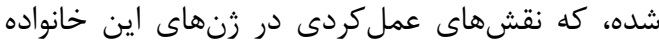

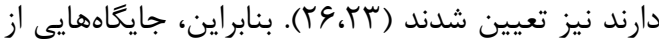

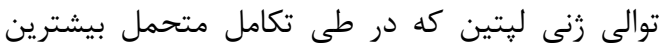

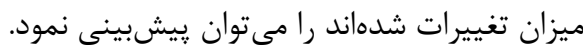
فيلوزنى و تعيين مسير تكاملى تئى

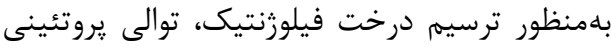

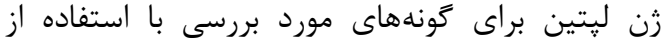

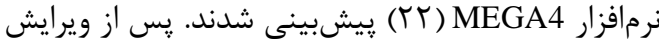

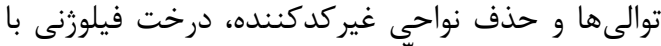

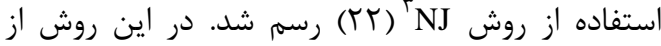

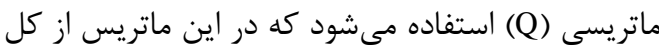

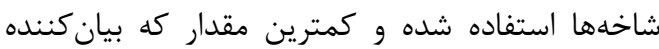

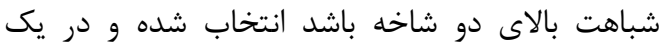

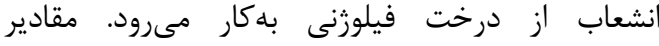

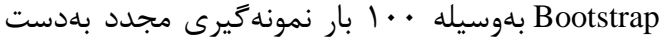

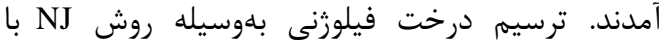
استفاده از رابطه ا انجام شد: Q(i, j) =(r-2)d(i, j) - رابطه (1) (1)

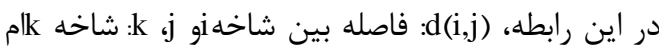

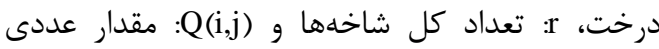

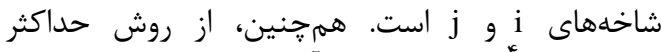

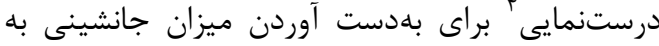

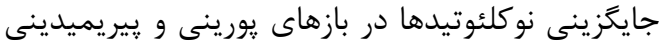

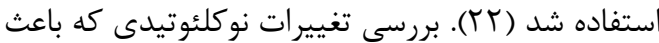

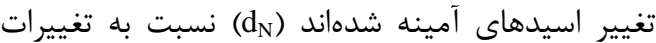
نوكلئوتيدى كه تأثيرى در اسيد آيد آمينه حاصله نداند نداشتهاند

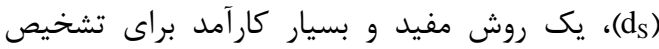

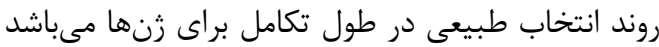

بنابراين، با استفاده از مقدار عددى اين نسبت

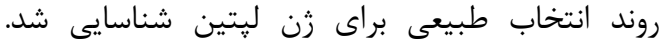

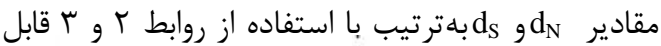

محاسبه هستند.

$\mathrm{d}_{\mathrm{N}}=-3 / 4 \operatorname{Ln}\left(1-4 / 3 \mathrm{P}_{\mathrm{N}}\right)$

2- Alignment

4- Maximum Composite Likelihood 
r). تجزيه فيلوزنى و تكامل مولكولى لهيت

يك روش مفيد و بسيار كارآمد براى تشخيص روند

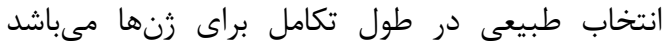

(1)
ذكر شده نيز صدق مى كند. همانطور كه ذكر شد، نتايج

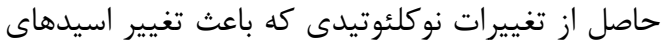

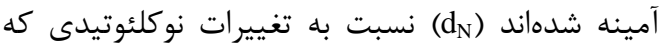

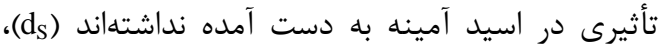
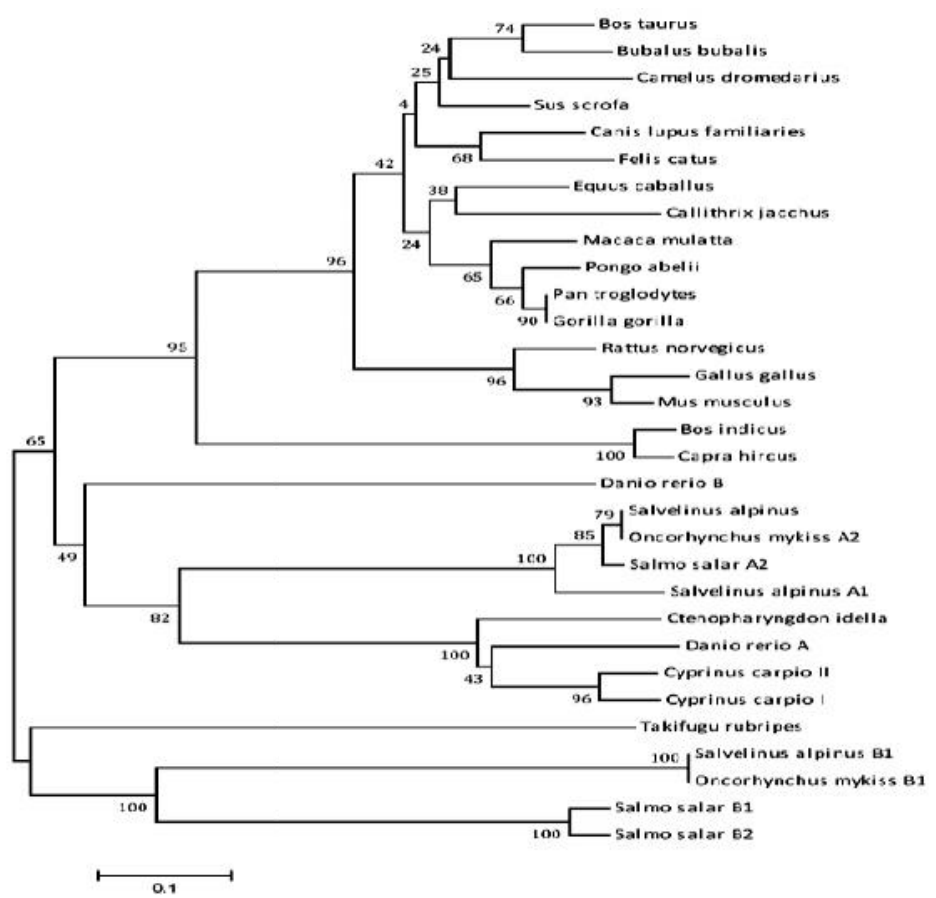

شكل ا- درخت فيلوزنتيك توالىهاى يروتئينى ليتين در گونهاى مورد بررسى ( / • نرخ جايكزينى اسيدهاى آمينه در هر جايغاه).

جديد و عمل كرد جديد براى اين زيروتئينها ميىشود.

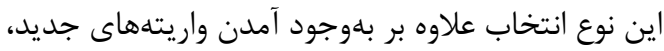

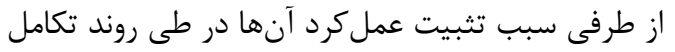

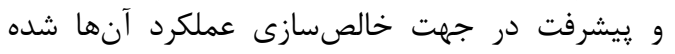

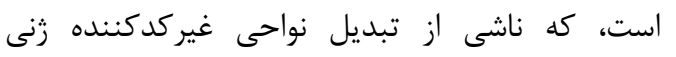

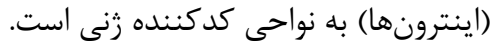

اگر اين نسبت بيشتر از يك باشد، انتخاب مثبت،

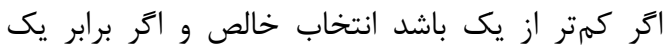

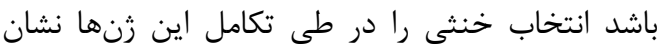

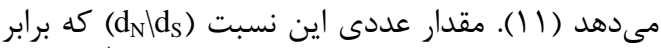

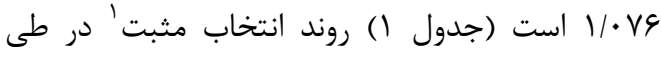

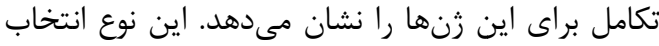

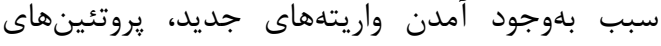

جدول ا- يارامترهاى تعيين روند انتخاب طبيعى در زن ليتين

\begin{tabular}{|c|c|c|}
\hline 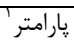 & مقدار عددى & انحراف استاندارد \\
\hline $\mathrm{d}_{\mathrm{N}}$ & $\cdot|A T|$ & $\cdot / \cdot \Delta V$ \\
\hline $\mathrm{d}_{\mathrm{s}}$ & . IVGY & . $1 \cdot \mu r$ \\
\hline $\mathrm{d}_{\mathrm{N}} / \mathrm{d}_{\mathrm{S}}$ & $1 / \cdot \sqrt{ } 9$ & שא \\
\hline
\end{tabular}


r نتايج حاصل از درصد جابهجايى نوكلئوتيدهاى زن رين

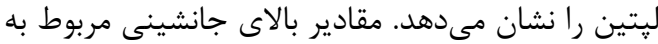

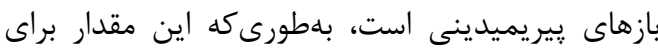

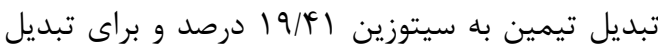

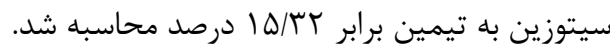

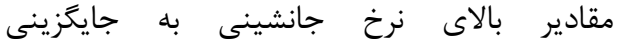

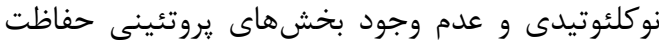

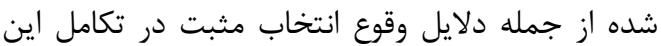

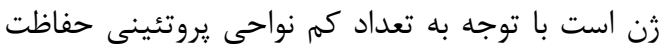

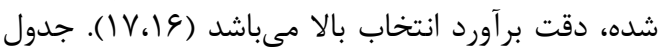

\begin{tabular}{|c|c|c|c|c|}
\hline نوكلئوتيد & A & $\mathrm{T}$ & $\mathrm{C}$ & $\mathrm{G}$ \\
\hline $\mathrm{A}$ & - & F/9 & $\Delta / \Lambda T$ & $I T / \cdot T$ \\
\hline $\mathrm{T}$ & $\Delta / \cdot V$ & - & $|9 / 4|$ & $4 / 19$ \\
\hline C & $\Delta / \cdot V$ & $1 Q / T T$ & - & 4/A9 \\
\hline G & $\mid T / F A$ & $y / 9$ & Q/AT & - \\
\hline
\end{tabular}

ليتين را تشكيل ميىدهد كه اين امر نشاندهنده

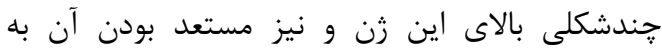

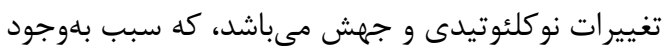

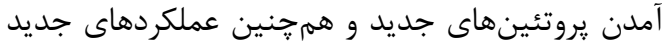

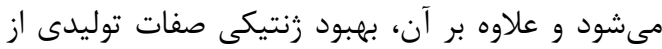

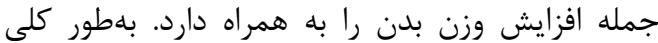

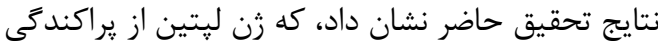

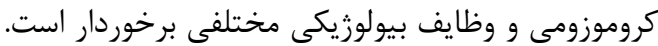

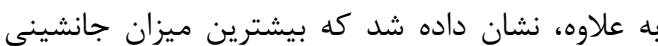

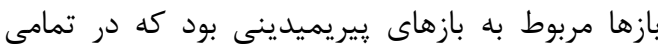

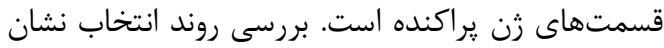

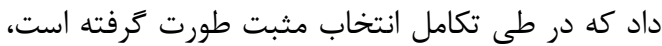

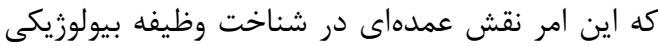

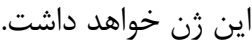

اين مقادير براى بازهاى : يورينى كمتر و بلهترتيب

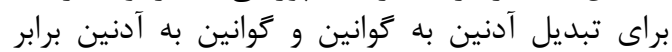

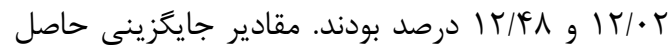

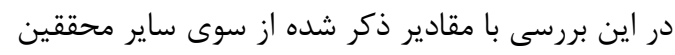

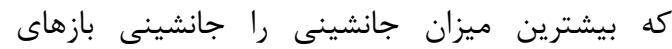

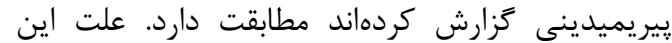

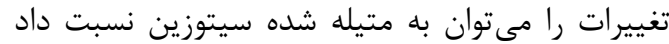

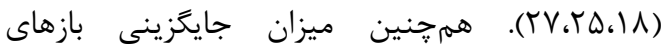

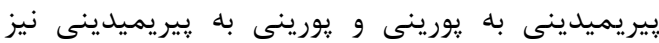

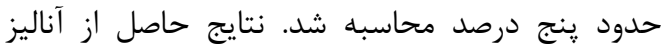

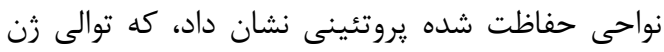

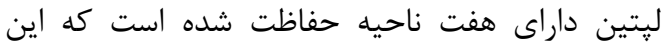

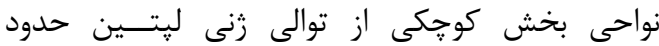

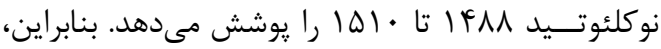

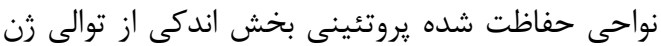

1. Ahima, R.S. 2005. Central actions of adipocyte hormones. Trends Endocrinology and Metabolis, 16: 307-313.

2. Ashwell, C.M., S.M. Czerwinski, D.M. Brocht and J.P. McMurtry. 1999. Hormonal regulation of leptin expression in broiler chickens. American Journal of Physiology, 276: 226-232.

3. Bado, A., S. Levasseur, S. Attoub, S. Kermorgant, J.P. Laigneau, M.N. Bortoluzzi, L. Moizo, T. Lehy, M. Guerre-Millo, Y. Le Marchand-Brustel and M.J. Lewin. 1998. The stomach is a source of leptin. Nature, 394: 790-793.

4. Caro, J.R., M.K. Sinha, J.W. Kolaczynski, P.L. Zhang and R.V. Considine. 1996. Leptin: The tale of an obese gene. Diabetes, 45: 1455-1462.

5. Chehab, F.F. 2000. Leptin as a regulator of adipose mass and reproduction. Trends Pharmacology Science, 21: 309-314.

6. Doyon, C., G. Drouin, V.L. Trudeau and T.W. Moon. 2001. Molecular Evolution of Leptin. General and Comparative Endocrinology, 124: 188-198.

7. Dridi, S., N. Raver, E.E. Gussakovsky, M. Derouet, M. Picard, A. Gertler and M. Taouis. 2000. Biological activities of recombinant chicken leptin $\mathrm{C} 4 \mathrm{~S}$ analog compared with unmodified leptins. Am Journal of Physiology, 279: 116-123.

8. Frederich, R.C., A.A. Hamann, S. Anderson, B. Lollmann, B.B. Lowell and J.S. Flier. 1995. Leptin levels reflect body lipid content in mice: Evidence for diet-induced resistance to leptin action. Nature Medicine Journal, 1: 1311-1314.

9. Hoggard, N., L. Hunter, J.S. Duncan, L.M. Williams, P. Trayhurn and J.G. Mercer. 1997. Leptin and leptin receptor mRNA and protein expression in the murine fetus and placenta. Proceedings of the National Academy of Science, USA, 94: 11073-11078.

10. Jin, L., B.G. Burguera, M.E. Couce, B.W. Scheithauer, J. Lamsan, N.L. Eberhardt, E. Kulig and R.V. Lloyd. 1999. Leptin and leptin receptor expression in normal and neoplastic human pituitary: 
rIT تجزيه فيلوزنى و تكامل مولكولى ليتين

Evidence of a regulatory role for leptin on pituitary cell proliferation. Journal of Clinical Endocrinology and Metabolism, 84: 2903-2911.

11. Li, W.H. 1997. Molecular Evolution. Sinauer, Sunderland, Mssachussets. USA.

12. Librado, P. and J. Rozas. 2009. DNASP v5: software for comprehensive analysis of DNA polymorphism data. Journal of Bioinformatics, 25: 1451-1452.

13. Masuzaki, H., Y. Ogawa, N. Sagawa, K. Hosoda, T. Matsumoto, H. Mise, H. Nishimura, Y. Yoshimasa, I. Tanaka, T. Mori and K. Nakao. 1997. Nonadipose tissue production of leptin: Leptin as a novel placenta-derived hormone in humans. Nature Medicine Journal, 3: 1029-1033.

14. Morash, B., A. Li, P.R. Murphy, M. Wilkinson and E. Ur. 1999. Leptin gene expression in the brain and pituitary gland. Endocrinology, 140: 5995-5998.

15. Nei, M. and S. Kumar. 2000. Molecular evolution and phylogenetics. New York: Oxford University Press. pp: 51-72.

16. Ngandu, N., K. Scheffler, P. Moore, Z. Woodman, D. Martin and C. Seoighe. 2008. Extensive purifying selection acting on synonymous sites in HIV-1 Group M sequences. Virology Journal, 5 : 160-171.

17. Phuphuakrat, A. and P. Auewarakul. 2003. Heterogeneity of HIV-1 Rev response element. AIDS Research and Human Retroviruses, 19: 569-574.

18. Picoult-Newberg, L., T.E. Ideker, M.G. Pohl, S.L. Taylor, M.A. Donaldson, D.A. Nickerson and M. Boyce-Jacino. 1999. Mining SNPs from EST Databases. Journal of Genome Research, 9: 167-174.

19. Saadoun, A. and B. Leclercq. 1983. Comparison of in vivo fatty acid synthesis of the genetically lean and fat chickens. Comparative Biochemistry and Physiology, 75: 641-644.

20. Senaris, R., T. Garcia-Caballero, X. Casabiell, R. Gallego, R. Castro, R.V. Considine, C. Dieguez and F.F. Casanueva. 1997. Synthesis of leptin in human placenta. Endocrinology, 138: 4501- 4504.

21. Sobhani, I., A. Bado, C. Vissuzaine, M. Buyse, S. Kermorgant, J.P. Laigneau, S. Attoub, T. Lehy, D. Henin, M. Mignon and M.J. Lewin. 2000. Leptin secretion and leptin receptor in the human stomach. Gut, 47: 178-183.

22. Tamura, K., J. Dudley, M. Nei and S. Kumar. 2007. MEGA4: Molecular Evolutionary Genetics Analysis (MEGA) Software Version 4.0. Journal of Molecular Biology and Evolution, 24: 1596-1599.

23. Taouis, M., J.W. Chen, C. Daviaud, J. Dupont, M. Derouet and J. Simon. 1998. Cloning the chicken leptin gene. Gene, 208: 239-242.

24. Tavaria, M., T. Gabriele, I. Kola and R.L. Anderson. 1996. A hitchhiker's guide to the human Hsp70 family. Cell stress and chaperones, 1: 23-28.

25. Vignal, A., D. Milan, M. San-Cristobal and A. Eggen. 2002. A Review on SNP and other Types of Molecular Markers and their use in animal Genetics. Journal of Genetic Selection and Evolution, 34: 275-305.

26. XiangLong, L., W. ZhaoLong, L. ZhengZhu, G. YuanFang, Z. RongYan and Z. GuiRu. 2006. SNP Identification and Analysis in Part of Intron 2 of Goat MSTN Gene and Variation within and among Species. Journal of Heredity, 97: 285-289.

27. Zhang, Y., R. Proenca, M. Maffei, M. Barone, L. Leopold and J.M. Friedman. 1994. Positional cloning of the mouse obese gene and its human analogue. Nature, 372: 425-432. 


\title{
Phylogenetic Analysis and Molecular Evolution of the Leptin Gene \\ (Short Paper)
}

Javad Ahmadpanah

Young Researchers and Elite Club, Islamic Azad University, Ilam Branch, Ilam, Iran

(Corresponding author: ajavad65@gmail.com)

Received: May 13, $2013 \quad$ Accepted: July 22, 2014

\begin{abstract}
In the current study, phylogenetic analysis and molecular evolution of the mammalian's Leptin was investigated. Data was achieved and aligned by searching its genome database, while all examined mammals contained only a single copy of the Leptin. The nucleotide substitution rate of the sequences and molecular evolution of the Leptin were calculated by maximum likelihood and neighbor-joining method respectively and phylogenetic tree was constructed. Bioinformatics researches results identified that Leptin genes are distributed across the genome. On the other hand base substitution rate of the pyrimidines to pyrimidines or purines is much more than that of in purines to pyrimidines or purines. The $d_{N} / d_{s}$ ratio of the Leptin sequences indicated that Positive selection was accrued during evolution which made new branches to give different responses. This can justifies high polymorphism of the Leptin. Phylogenetic analysis showed that Leptin proteins based on mainly are divided to two clades their evolutionary relationships. In the first clade the protein sequences are divided to mammals and chicken and in the second clade called the others we have all types of fish.
\end{abstract}

Keywords: Leptin, Mammals, Natural Selection, Phylogeny 